\title{
NANOSCALE ETCHING AND INDENTATION OF SILICON(001) SURFACE WITH CARBON NANOTUBE TIPS.
}

\author{
Submitted to Physical Review Letters by \\ Fedor N. Dzegilenko, Deepak Srivastava, and Subhash Saini \\ NASA Arnes Research Center, Moffett Field, CA 94035 \\ PACS numbers: $61.48 .+\mathrm{c}, 81.65 . \mathrm{Cf}, 07.79 . \mathrm{Lh}$
}

July 10,1998

\begin{abstract}
The possibility of nanoscale etching and indentation of $\operatorname{Si}\{001\}(2 \times 1)$ surface by $(8,0)$ and $(10,10)$ carbon nanotule tips is demonstrated, for the first time, by classical molecular dynamics simulations employing Tersoff's many-body potential for a mixed C/Si/Ge system. In the nanotube tip barely touching the surface scenario atomistic etching is observed, where as in the nanoindentition scenario nanotube tip penetrates the surface without much hindrance. The results are explained in terms of the relative strength of $\mathrm{C}-\mathrm{C}, \mathrm{C}-\mathrm{Si}$, and $\mathrm{Si}-\mathrm{Si}$ bonds.
\end{abstract}

Lithography plays a key role in semiconductor manufacturing. Currently surface patterning is achieved by means of optical lithographic techniques. However, with the industry moving towards the fabrication of semiconductor devices with the size features of $100 \mathrm{~nm}$ and less, [1] the ability of even deep ultraviolet light $(193 \mathrm{~nm})$ sources will be exhausted due to the absence of optically transparent materials at wavelengths significantly shorter than 193 $\mathrm{nm}$. The technological community, thus, is actively searching for alternative approaches to materials fabrication at nanoscale. Among several alternatives to optical lithography are the utilization of high-resolution laser direct writing to structure silicon on the nanometer scale, [2] electron beam lithography, [3] and proximal probe lithography. [4]

The proximal probe tips have been shown to be a powerful tool for the manipulation of individual atoms and molecules at the nanometer scale. [5-9] A main difficulty faced in proximal probe lithographic techniques has been that the probe metallic tips often break following direct collision with the solid surface. Other alternatives to the metallic tips have been explored recently. Carbon nanotubes have been mounted on top of the silicon cantilever $[10]$ to be used as proximal probe tips. The carbon nanotubes are rolled-up sheet of graphene made of six folded benzene-type rings of carbon. The nanotubes are both of single and multiwall (concentric cylindrical shells) nature and have unique elastomechanical properties. [11] These are the strongest material known along the axial direction and yet are highly elastic and flexible along the radial direction.

Several groups have untertaken atomistic simulations of tip-surface interactions using models of usual metallic ancl/or diamond tips [12-15] interacting with diamond and/or silicon surfaces. In a recent study, [16] we have simulated the possibility of selective etching of diamond surface by using a carbon nanotube tip chenically modified with a $\mathrm{C}_{2}$ species 
attached to the end cap of the tip. A single dimer of carbon atoms etched out of the surface by the tip, with the main result that the strongly borded $\mathrm{C}_{2}$ chemical species on the tip is able to etch a weakly bonded $\mathrm{C}_{2}$ dimer from the surlace. This raises the possibility that one can perhaps use a bart nanotube tip, made of strong $\mathrm{C}-\mathrm{C}$ covalent bonds, for nanolithography or atomistic et hing on comparatively weaker bonded semiconductor surfaces such as silicon and/or germanium.

Using classical molecular dynamics (MD) simulations with Tersoff;s potential for Si-C, $\mathrm{Si}-\mathrm{Si}$, and $\mathrm{C}-\mathrm{C}$ interactions, $[17]$ in this paper we investigated the possibility of bare nanotubeetching and -indentation on silicon surfaces. The etching and indentation parameters are entirely determined by the mechanical properties of the tips and by the underlying tip-surface chemical reactions. Much of the complications involved in controlling the electronic behavior of the tip may be avoided. 'The possibility to pick up a single atom in field-free SPM regime has been recently experimentally demonstrated by Dujardin et al. [18] Single Ge atoms were extracted in a controlled nanner from the Ge(111) surface by the "traditional" SPM tip made of Ge atoms in a zero bias operation. Using MI) simulations we show, for the first time, that similar atomic scale etching of $\mathrm{Si}$ surfaces is possible with carbon nanotube tips under zero bias operation conditions. The advantage of nanotube tip over the traditional metal or semiconductor tip would be that the nanotube tip can survive later in experiments.

The single-height-stepped $\mathrm{Si}\{001\}(2 \times 1)$ surface has been chosen to model the surface of silicon. The slab representing the surface is composed of 15 full layers containing 128 atoms each and half-filled top layer representing the upper terrace of the stepped surface. The total number of atoms is 1984 . The $(8,0)$ carbon nanotube (composed of 222 carbon atoms) and larger diameter $(10,10)$ carbon nanotube (composed of 470 carbon atoms) are used as tips as shown in Figures 1a and 2a, respectively. The caps of these two nanotube tips have different curvature and, as a result, the carbon atoms of the tip are expected to exhibit different chemical reactivity. Three different surface sites are considered. Those are the upper-terrace and lower terrace dimer on top sites and the dimer site at the step-edge. The dimer rows for lower and upper terrace are perpendicular to each other. The inclusion of step edge site allows to compare the reactive dynamics of the flat surface with the one near the step edge.

Tersoff's many-body empirical interatomic potential for multicomponent $\mathrm{Si} / \mathrm{C} / \mathrm{Ge}$ system has been used to describe the $\mathrm{C}-\mathrm{C}$ and $\mathrm{C}-\mathrm{Si}$ interactions. [17] This is a reactive potential which allows for a description of bond formation and rupture. The bottom two layers of the silicon surface are held ixed. Next two layers are subjected to frictional and dissipative Langevin forces to drain out the excess kinetic energy and to hold constant the temperature of the system. The top two layers of the open end of carbon nanotubes are also constrained to vertically move the nanotule in a predetermined manner. Classical equations of motion are integrated using a third-order predictor-corrector method with variable time steps. Periodic boundary conditions are applied in the surface plane. Both interacting systems are initially equilibrated to $300 \mathrm{~K}$.

The nanotube vertical motion is achieved by displicing the constrained atoms at each (variable) time step in the desired manner. All the trajectories start with the distance between the specified surfac: dimers and the closest (to the surface) atom of the nanotube cap

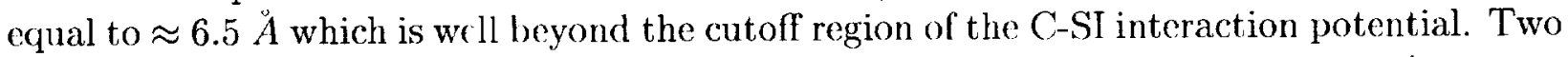
regimes of nanotube tip operation are considered. In the first scenario the nanotubes move 
down and barely touch the surface mimicking the nanotube-surface chemical reaction induced by the vertical proximity of the nanotube and the surface. This is accomplished by downward displacement of the nanotube in 5000 variable time steps with $0.0013 \dot{A}$ displacement per step. In the absence of nanotube-surface interactions this approach velocity would place the nanotube directly on top of the surface dimers. The system is then equilibrated for 5000 additional steps and the nanotube is then pulled back. The nanotube displacement during the retraction phase is varied to slower velocities to ensure the convergence of the results. The per time-step retraction displacement of 5 times slower, than the value on the way down, is found to be the most computationally efficient without affecting the outcome of the simulations.

For the $(8,0)$ nanotube, the selective removal of the surface dimer occurs with minimum disturbance of the underlying silicon surface (Fig. 1b). The observed transition state configuration is linear. After a series of bond rearrangements, the atoms withdrawn from the surface are adsorbed on two neighbouring carbon atoms of the outer pentagon of the nanotube cap as shown in Fig. 1c. For the larger diameler $(10,10)$ nanotube, the analogous simulations result in the removal of three silicon atoms from the surface (Fig. 2b,c) via the distorted four member ring transition state. Similar to the previous case, the surface remains virtually undisturbed after the completion of mechano-chemical nanotube-surface reaction (Fig. 2c). Qualitatively similar results are observed for the lower terrace and step edge surface sites, though somewhat larger disturbance of the substrate is observed in the latter case.

In the second scenario, the nanotubes are pushed into the surface to make "nano-holes". This "digging" scenario allows the study of indentation phenomenon. Starting with the

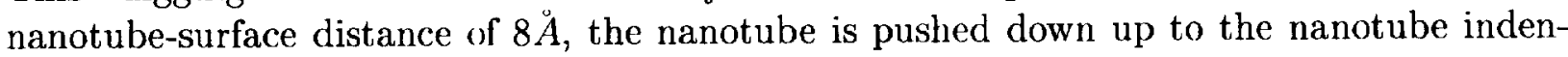
tation depth of $4 \breve{A}$ into the surface slab. The nanotube is then retracted similarly to the previous case.

The results of simulations when the $(8,0)$ nanotube is allowed to penetrate inside the silicon slab are discussed next. Figure 3 a shows the instance when the nanotube has indented and penetrated the silicon surface. The nanotube appears to completely preserve its original shape. The retraction of the nanotube causes adsorption of both the surface and bulk silicon atoms at the nanotube tip. At the end of the simulations, the internal structure of the silicon surface is strongly altered in the interaction region (Fig. 3b) though no apparent "hole" is formed due to the "healing" thermal motion of bulk surface atoms. Simulations with the substrate temperature reduced to $50 \mathrm{~K}$ produced similar results. The main conclusion is that the indentation and penetration of silicon surface occurs easily and can be compared with the process of a knife cutting the butter. To compare the above with the contrasting behavior of the nanotube indentation on a harder diamond $\{001\}(2 \times 1)$ surface, the results are shown in Fig. 3c. In the nanotube indentation on the diamond surface, significant deformation of the nanotube is observed (Fig. 3c) and the diamond surface is not indented at all.

In order to gain a bette: understanding of the details of the reactive dynamics, we have calculated vertical forces experienced by the tube and surface atoms during the nanotubesurface interaction period. The representative plots in the touching scenario (Figures $4 a, b)$ show the vertical force on the nanotube atoms vs. the displacement of $(8,0)$ nanotube towards the silicon surface. Zero of the $\mathrm{x}$-axis corresponds to the initial vacuum nanotube-surface distance of $6.5 \AA$. The nar otube-surface distance varies in a complex manner and is not 
shown here. The surface layer position in the absence of nanotube-surface interaction is shown by arrows in the figu e. Two curves shown in Fig. 4a correspond to the vertical force on the lowest two atoms of the nanotube tip, while those shown in Fig. $4 \mathrm{~b}$ correspond to the forces on two silicon atoms of the upper-terrace surface dimer. No significant vertical forces are experienced by these four atoms until the nanotube displacement reaches $4 \vec{A}$

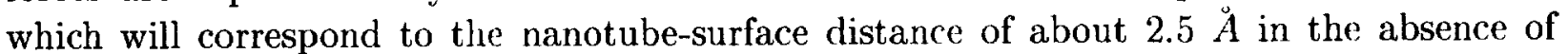
nanotube-surface interactio:. At this moment, the sharp fluctuations of the vertical force are observed simultaneously for one of the nanotube atoms and for one of the surface silicon atoms indicating the formation of the first C-Si chemical bond. The formation of the second $\mathrm{C}-\mathrm{Si}$ bond occurs later at the nanotube displacement of $\approx 4.7 \AA$ indicating a sequential mechanism of the nanotube binding to the surface. Subsequent dynamics is dominated by a series of bond rearrangements and the atoms eventually lifted from the surface by the tip are shown in Fig 1c.

Figures 5 a,b show the trital vertical force experienced by $(8,0)$ nanotube versus the nanotube displacement towards the silicon and diamond surfaces respectively. Zero of the $\mathrm{x}$-axis

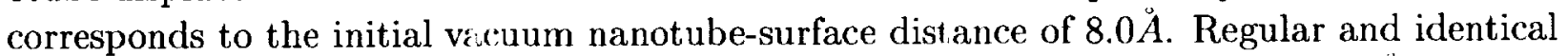

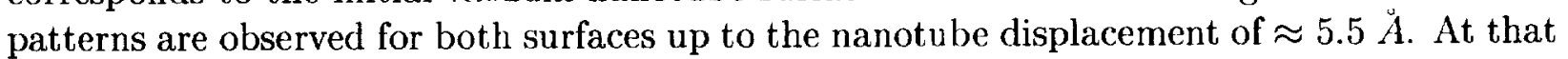
moment significant fluctuations of the force start to appear as a result of direct chemical interaction with the surfaces. Both the variation and the magnitude of the force are significantly larger for the nanotube interacting with the diamond surface. This is consistent with the difference in the simulation outcomes for the silicon and diamond surfaces (indentation vs. compression, Fig. 3a-c).

The effects of the long range van der Waals interactions on the above dynamics were also studied. Presently, there ar no van der Waals interaction parameters tested with Tersoff's heteroatomic $\mathrm{Si} / \mathrm{Ge} / \mathrm{C}$ potential. To estimate the van der Waals repulsion on the nanotube as it approaches the surface, we considered the nanotube interaction with the diamond surface including van der Waals C-C interaction parameters incorporated into the Tersoff-Brenner reactive potential. [15] The system is equilibrated first and then total energy minimization is performed at a variety of fixed nanotube-surface distances. The van der Waals interaction potential from these calculations is slightly attractive all the way down to $\approx 1 \AA$ where the repulsion kicks in. Thus, the long range forces do not introduce any barrier in the incoming channel and the van der Waals repulsion starts after the stronger chemical interaction is already in effect. For the nanotube-silicon surface, the effect of long-range interactions is expected to be even smaller than for the nanotube-diamond surface because the interatomic distances within the silicon crystal are $\approx 1.5$ times larger than those of the diamond. Thus, we infer that the inclusion (f long-range interactions will not significantly alter the reported results.

The observed results are qualitatively accounted for by the relative strength of interatomic bonds for the Si-C system. F'or the silicon surface, the interatomic Si-Si bonds within a silicon crystal are weaker than the corresponding $\mathrm{Si}-\mathrm{C}$ bonds resulting in removal of silicon atoms off the silicon surface (Fig. 1,2). At the same time, the C-C bonds which hold the nanotube together are stronger than both the $\mathrm{Si}-\mathrm{C}$ and $\mathrm{Si}-\mathrm{Si}$ bonds resulting in easy indentation of silicon surface by the nanotube (Fig. 3) with the nanotube preserving its original shape. The situation is quite the spposite for the diamond surface. The hierarchy of $\mathrm{C}-\mathrm{C}$ bond strengths for the diamond-carbon nanotube system is the following: the strongest one is 
the $\mathrm{C}-\mathrm{C}$ bond within a diamond crystal, the $\mathrm{C}-\mathrm{C}$ diamond-nanotube bond is the next, and $\mathrm{C}-\mathrm{C}$ bond inside the nanotube is the weakest one. This trend explains both the observed compression of the nanotube (Fig. 3) and the cleavage of the internanotube bonds observed in the "touching" scenario. Our results indicate that both the $(8,0)$ and $(10,10)$ nanotubes are able to extract silicon atoms off the silicon surface indicating the relative unimportance of the nanotube size and the curvature of its cap on the simulation outcome in the gentle "touching" regime. The observed transition states are either linear or four-member ring type meaning that either one or two lowest atoms of the nanotube cap are involved in chemical bonds formation with the surface atoms irrespective of the nanotube size.

In conclusion, we have demonstrated through classical MD simulations the possibility of selective etching and indentation of silicon surfaces by carbon nanotubes mounted on an scanning probe microscope cantilever. The proposed method is very robust and does not require applied voltage between the nanotube tips and the surface. The simulation results are timely in the sense that external voltage assisted nanotube-nanolithography [19] and arrays of "traditional" SPM tips in parallel [20] and tunnel-free [18] regimes have been recently demonstrated in experiments. The proposed method of etching of silicon surface combines the advantages of the above methods and provides additional input to the enabling technology for the next generation of SPM based lithographic techniques.

This work was supported by MRJ,Inc. under NASA contract NAS2-14303. 


\section{References}

[1] The National Technology Roadmap for Semiconductors SIA, San-Jose (1997).

[2] M. Müllenborn, in Frontiers in Nanoscale Science of Micron/Submicron Devices, A-K Jauno, E. V. Buzaneva, editors (Kluwer Academic Publishers, Dordrecht. p 85. 1986).

[3] T. H. P Chang, D. P.Kern, E. Kratschmer, K.Y. Lee, H. E. Luhn, M.A. McCord, S. A. Rishton, and Y. Vladimirsky, IBM J. of Res. Develop. 32, 46 (1988).

[4] A. L. de Lozanne, W. F. Smith, and E. E. Ehrichs Technology of Proximal Probe Lithography (Bellingham: SPIE Optical Engineering) p. 188 (1993).

[5] J.K. Gimzewski, T. A. Jung, M. T. Cuberes, and R. R. Schlitter, Surf. Sci. 386, 101 (1997); H. Tang, M. T. Cuberes, C. Joachim, and J. K. Gimzewski ibid 115; T. A. Jung, R. R. Schlittler, J. K. Gimzewski, H. Tang, C. Joachim, Science 271, 181 (1996).

[6] M. F. Crommie, C. P. I utz, and D. M. Eigler, Science 262, 218 (1993).

[7] Ph. Avouris, I-W Lyo, and Y. Hasegawa, J. Vac. Sci. Technol. A 11, 1725 (1993); I-W Luo and Ph. Avouris Science 253, 173 (1991); T-C, Chen, C. Wang, G. C. Abeln, J. R. Tucker, J. W. Lyding, Ph. Avouris, R. E. Walkup, Science 1590 (1995).

[8] D. H. Huang and M. Aono, Surf. Sci. 386, 166 (1997).

[9] T. Komeda, R. Hasunuma, H. Mukaida, H. Tokumoto, Surf. Sci. 377-379, 149 (1997).

[10] H. Dai, J. H. Hafner, A. G. Rinzler, D. T. Colbert, and R. E. Smalley Nature 384, 147 (1996).

[11] B. I. Yakobson, C. J. Brabec, and J. Bernholc, Phys. Rev. Lett. 76, 2511 (1996); D. Srivastava and S. Barnard, Supercomputing (1997).

[12] J. S. Kallman, W. G. Hoover, C. G. Hoover, A. J. DeGroot, S M. Lee, and F. Wooten Phys. Rev. B 47, 3318 (1993).

[13] U. Landman, W. D. Luedtke, N. A. Burnham, and R. J. Colton Science 248, 454 (1990); H. Raffi-Tabor, J. B. Pethica, and A. P. Sutton Mater. Res. Soc. Symp. Proc. 239, 313 (1992); O. Tomagnini, F. Ercolessi and E. Tosatti Surf. Sci. 287/288, 1041 (1993).

[14] J. A. Harrison, C. T. White, R. J. Colton, and D. W. Brenner 1992 Surf. Sci. 271, 57 (1992); S. B. Sinnott, R. J. Colton, C. T. White, and D. W. Brenner Surf. Sci. 316, L1055 (1994).

[15] D. W. Brenner, S. B. Sinnott, J. A. Harrison, O. A. Shenderova, Nanotechnology 7, 161 (1996).

[16] F.N. Dzegilenko, D. Srivastava, S. Saini, Nanotechnology,in press (1998).

[17] J. Tersoff, Phys. Rev I3 39, 5566 (1989). 
[18] G. Dujardin, A. Maine, O. Robert, F. Rose, C. Joachim, and H. Tang, Phys. Rev. Lett. 80, 3085 (1998); P. Molinas-Mata, A. Mayne, and G. Dejardin, Phys. Rev. Lett. 80, 3101 (1998).

[19] H. Dai, private communication.

[20] S. C. Minne, S. R. Manalis, A. Atalar, C. F. Quate, J. Vac. Sci. Technol. B 14, 2456 (1996); C. F. Quate, Surf. Sci. 386, 259 (1997) and references therein. 